\title{
Contabilidade mental e mudanças em preços: um estudo experimental Mental accounting and changes in prices: an experimental study
}

\author{
Marcos Gonçalves Ávila \\ marcos@coppead.ufrj.br \\ Professor Associado do Instituto Coppead de Administração, Universidade Federal do Rio de Janeiro \\ Rachael Botelho Figueiredo \\ rachael@ashoka.org.br \\ Mestre em Administração - Instituto Coppead de Administração, Universidade Federal do Rio de Janeiro
}

Editor Científico Responsável: Prof. Dr. José Márcio de Castro Professor do Programa de Pós-graduação em Administração da Pontifícia Universidade Católica de Minas

\begin{abstract}
Resumo
Neste trabalho testaram-se, por meio de um experimento, hipóteses relativas à operação dos princípios de contabilidade mental no contexto de informações comparativas de preços. Os resultados do experimento mostram que a inclusão de preço de referência nas informações comparativas de preços e a maneira de apresentar os ganhos e as perdas em relação ao preço de referência - de forma absoluta (em reais) ou de forma relativa (percentuais) - podem influenciar percepções. Os resultados sugerem ainda que consumidores de baixa escolaridade são especialmente suscetíveis aos efeitos de diferentes maneiras de apresentar informações de preço.
\end{abstract}

Palavras-chave: Contabilidade mental. Teoria dos prospectos. Experimentos econômicos.

\begin{abstract}
This experimental study tested hypotheses about the operations related to the principles of mental accounting. The experimental results provided evidence that both the inclusion of a reference price on the set of comparative pricing information and the manner by which gains and losses are presented influence price perceptions. The results also suggest that lower income consumers are especially influenced by different frames.
\end{abstract}

Key words: Mental accounting, Prospect Theory, Experimental economics 


\section{INTRODUÇÃO}

Thaler (1985) cunhou a expressão contabilidade mental para se referir ao conjunto de operações cognitivas que as pessoas fazem para organizar, analisar e acompanhar as atividades financeiras nas quais se envolvem. Uma questão de interesse nas pesquisas sobre o tema se refere à maneira como as pessoas combinam dois ou mais eventos financeiros dentro de uma mesma conta mental. Thaler (1985), por exemplo, apresentou o seguinte problema a 87 estudantes de uma universidade norte-americana:

Quem você preferiria ser no cenário a seguir: O Sr. A ganha \$50 na loteria estadual ao passo que o Sr. B ganha $\$ 100$ na loteria federal, mas inesperadamente passa a dever $\$ 50$ a seu locador por ter manchado o carpete.

A previsão foi de que a maioria dos estudantes preferiria ser o Sr. A, o que de fato aconteceu. Segundo Thaler (1985), em um cenário com dois eventos, em que um dos eventos é uma perda e o outro um ganho de maior valor, as pessoas se sentirão melhor se avaliarem os eventos de forma integrada, isto é, pelo ganho líquido (a forma integrada é ilustrada pela situação do Sr. A). A alternativa seria a de avaliar os eventos de forma separada (ilustrada pela situação do Sr. B).

A previsão de Thaler nesse estudo fundamenta-se em dois princípios da teoria dos prospectos (KAHNEMAN; TVERSKY, 1979). O primeiro princípio é o da satisfação marginal decrescente; por exemplo, dois ganhos separados de \$10 implicam uma satisfação maior do que um único ganho de \$20. O segundo princípio é o de que perdas são sentidas de forma mais intensa do que ganhos (por exemplo, perder \$10 acarreta uma sensação negativa mais intensa do que a sensação positiva de ganhar \$10). Kahneman e Tversky (1992) denominaram esse princípio de aversão a perdas e sugerem, com base em diversos estudos empíricos, que o coeficiente de aversão a perdas é de 2,5. Em outras palavras, a insatisfação de perder \$X é, em geral, 2,5 vezes maior do que a satisfação de receber $\$ X$. Experiências passadas negativas criam barreira para transações comerciais, confirmando a teoria da aversão à perda (ZHANG; FISHBACH, 2005).

Heath, Chatterjee e France (1995), a seu turno, discutem os princípios de operação da contabilidade mental no contexto de promoção de preços. Os autores descrevem o seguinte cenário: 
cadeira. Na loja, ele descobre o seguinte: o preço da cadeira foi reduzido de $\$ 300$ para $\$ 200$ e o preço da poltrona foi aumentado de $\$ 1.000$ para $\$ 1.050$.

Com relação aos preços originais, o comprador desse cenário fictício encara um ganho de \$100 e uma perda de \$50. Mentalmente, ele pode avaliar o cenário de duas maneiras: um ganho líquido de \$50 (avaliação que integra os dois eventos em um único evento) ou um ganho de \$100 e uma perda de \$50 (avaliação que mantém os dois eventos mentalmente separados). Com base na teoria dos prospectos, Thaler (1985) sugere que a avaliação integrada levará o consumidor a manifestar um maior grau de satisfação com a transação.

Heath, Chatterjee e France (1995) argumentam que o cenário em questão apresenta, entretanto, uma diferença importante em relação aos cenários testados nas pesquisas sobre contabilidade mental. Essa diferença se refere à inclusão de um preço de referência nas informações. A inclusão do preço de referência pode fazer diferença porque cria a possibilidade de o consumidor avaliar as alterações de preço em termos relativos. A situação poderia ser vista como um ganho de 33\% (a redução de \$300 para \$200) e uma perda de 5\% (o aumento de $\$ 1.000$ para $\$ 1.050$ ) ou como um ganho líquido de 3,8\%.

O princípio da satisfação marginal decrescente (cf. KAHNEMAN; TVERSKY, 1979; THALER, 1999) sugere que as pessoas tendem a pensar em termos relativos e, portanto, justifica-se a hipótese de que a avaliação do cenário é feita em termos percentuais. Com base na proposta de que o coeficiente de aversão a perdas é de 2,5, Heath, Chatterjee e France (1995) argumentam que, se a avaliação em termos relativos for adotada, a opção de visualizar os eventos de forma separada levaria a uma maior satisfação do que a avaliação integrada. As duas maneiras de apresentar a situação - forma relativa (em percentual) e forma absoluta (em valores monetários) - são financeiramente equivalentes, mas, conforme a discussão sobre contabilidade mental sugere, podem levar a percepções diferentes. Mensagens de marketing que apresentam o desconto de forma absoluta são mais eficientes quando o produto tem alto valor; em contrapartida, para produtos baratos o melhor é apresentar o desconto em termos percentuais (GENDALL et al., 2006).

O objetivo deste trabalho é testar um conjunto de hipóteses relativas à robustez dos princípios da contabilidade mental no contexto de cenários relativos a reduções e aumentos de preços. O estudo replica as pesquisas de Heath, Chatterjee e France (1995) no contexto brasileiro e propõe-se a avaliar a validade multicultural das propostas associadas à teoria dos 
prospectos e da contabilidade mental. Em visita ao Brasil, após receber o Prêmio Nobel de Economia de 2002, Daniel Kahneman declarou em entrevista à revista IstoÉ Dinheiro (FREITAS Jr., 2007):

\begin{abstract}
Nós realmente não sabemos com certeza [sobre a validade fora dos Estados Unidos dos resultados das pesquisas sobre a teoria dos prospectos]. Não há uma quantidade grande de estudos internacionais nessa área. Mas, pelo que observamos em algumas pesquisas, nossas principais descobertas são, provavelmente, bastante universais. É claro que também existem muitas diferenças culturais. Mas ainda não foi estudado e qualificado o peso dessas diferenças nas decisões feitas em experimentos iguais aos nossos em outros países. Aquilo que nós aprendemos vem dos Estados Unidos.
\end{abstract}

Este estudo, entretanto, vai além da replicação de estudos anteriores e estende a pesquisa para incluir nível de escolaridade como uma variável que pode mediar o impacto da maneira de apresentar informações comparativas de preço sobre as percepções do consumidor. A hipótese de que essa variável pode ser relevante nas pesquisas sobre contabilidade mental surgiu durante a fase-piloto do experimento, conforme será discutido adiante.

\title{
2 REVISÃO DA LITERATURA
}

\subsection{Teoria dos prospectos}

A teoria dos prospectos foi articulada por Kahneman e Tversky (1979). Eles reconhecem, seguindo a tradição das pesquisas de Herbert Simon, Prêmio Nobel de Economia de 1978, que dificuldades cognitivas têm grande influência no comportamento decisório "quando, diante de um problema complexo, as pessoas usam atalhos computacionais e operações de edição" (KAHNEMAN; TVERSKY, 1992, p. 298) - e mapeiam esses atalhos e suas consequências no comportamento decisório.

A articulação da teoria dos prospectos foi precedida de uma série de pesquisas empíricas que evidenciou ser possível mudar uma preferência entre alternativas de uma decisão em razão de alterações na maneira de apresentar o problema. Tversky e Kahneman 
(1981) usaram a expressão efeito framing para se referir a esse fenômeno. Para explicá-lo, a teoria dos prospectos distingue duas fases no processo decisório individual sob incerteza: uma etapa inicial de edição do problema, da qual resulta uma percepção do tomador de decisão sobre o problema e uma etapa subsequente de avaliação. A etapa inicial cumpre a tarefa de reduzir a complexidade do problema e obedece a um conjunto de operações de edição:

a) as pessoas percebem os resultados da decisão como ganhos ou perdas em relação a um ponto de referência neutro, que é assumido para a situação em questão;

b) se o ponto de referência adotado é tal que os resultados são percebidos como ganhos, então uma posição de aversão ao risco predomina. Caso contrário, (ponto de referência adota implica percepções de resultados como perdas) predomina uma atitude de aversão ao risco;

c) ganhos ou perdas relativas a esse ponto de referência são avaliados não por seu valor objetivo, mas por um valor subjetivo e pessoal denominado de utilidade. Essa noção de utilidade representa uma medida do grau de satisfação associado a cada resultado objetivo da decisão;

d) a relação entre o ganho ou a perda e a utilidade atribuída ao resultado é tal que as pessoas são mais sensíveis a diferenças entre resultados quando estes estão perto do ponto de referência. Em outras palavras, a diferença, em valores subjetivos, entre ganhos de \$10 e \$20 é percebida como maior do que a diferença entre ganhos entre \$110 e \$120. Essa operação cognitiva acarreta o que na literatura ficou denominado de sensibilidade marginal decrescente (THALER, 1999);

e) a intensidade da sensação associada à perda de um determinado montante é maior que a intensidade associada a um ganho do mesmo montante (o princípio da aversão a perdas). O coeficiente de aversão a perdas foi calculado em 2,5 , isto é, a sensação negativa associada a perda de um determinado valor é 2,5 vezes maior do que a sensação positiva associada a um ganho do mesmo valor.

Definindo preço de referência como aquele que o comprador adota como base para avaliar o preço efetivamente cobrado pelo produto, Serpa e Avila (2004) confirmaram a importância do efeito framing mostrando que diferentes formas de apresentar informações de preço têm grande impacto na decisão de compra. Thomas e Menon (2007) sugerem que consumidores menos confiantes tendem a ter um preço de referência mais alto do que os consumidores confiantes. 
A Figura 1 sumariza graficamente as propostas da teoria dos prospectos associadas à noção de ponto de referência. A função mostra, no eixo horizontal, os resultados da decisão como desvios do ponto de referência e associa, no eixo vertical, um valor subjetivo a cada resultado. Seu formato em S faz com que seja côncava para ganhos, de forma que cada unidade extra que seja ganha adiciona menos valor (utilidade) do que a precedente. No campo das perdas, a função é convexa, de forma que cada unidade perdida causa uma mudança menor no valor (utilidade) do que a precedente. A curva é mais íngreme do lado de perdas, de maneira a incorporar a noção de que o ser humano é mais sensível a perdas do que a ganhos de mesmo valor.

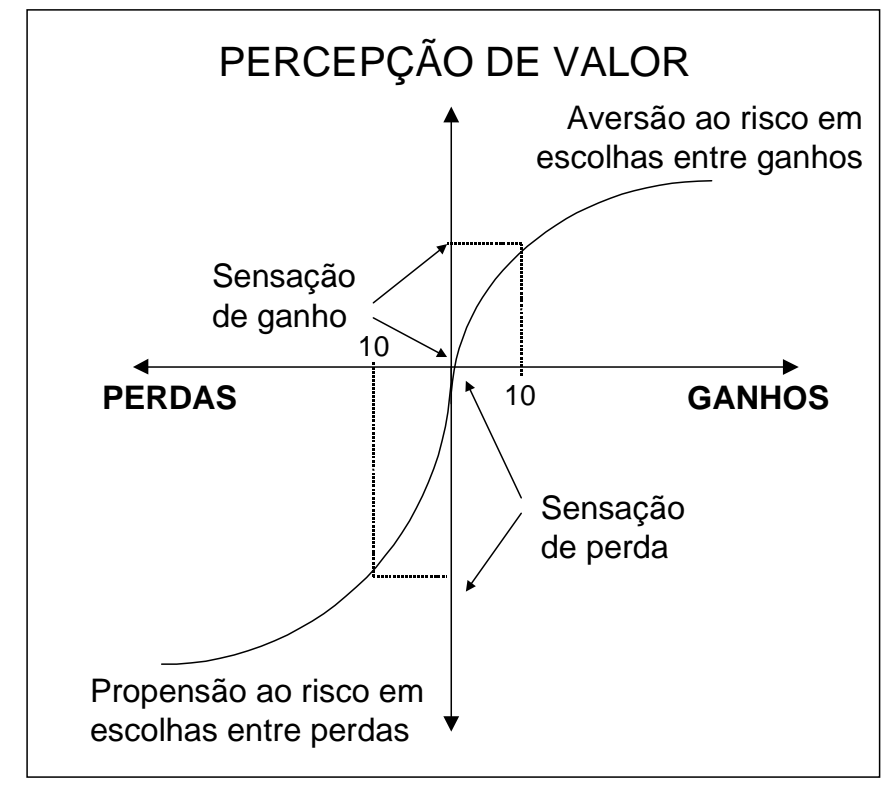

FIGURA 1: Representação gráfica da teoria dos prospectos Fonte: KAHNEMAN e TVERSKY, 1984.

A curva em $S$ da função-valor equivale à denominada lei Weber-Fechner, para a qual o montante que um estímulo precisa ser aumentado (ou diminuído) para que a diferença seja notada é proporcional ao valor do estímulo de referência (DAWES, 1988). A teoria dos prospectos sugere, portanto, conforme assinalou Russo e Schoemaker (1989, p. 28), que "as pessoas tendem a pensar sobre economias em termos percentuais e não em dólares absolutos", isto é, informações de ganhos ou perdas são processadas em termos relativos.

A teoria dos prospectos e o efeito framing foram objetos de diversos estudos (ODEAN, 1998; CAMERER, 2000; FENNEMA; PERKINS, 2008). Para campanhas de marketing 
relativas a comportamentos relacionados à saúde, por exemplo, a teoria dos prospectos é tida por Jones (2007) como mais efetiva do que a tradicional teoria econômica da utilidade.

\subsection{Contabilidade mental}

Com base na teoria dos prospectos, Thaler (1985) propõe e testa alguns princípios de operação da contabilidade mental que, se adotados, maximizariam a utilidade de alguém que se confronta com uma situação decisória envolvendo múltiplos eventos financeiros. Um desses princípios se refere a cenários envolvendo ganhos e perdas. Thaler demonstra que integrar perdas menores a ganhos maiores na contabilização mental do cenário é preferível a manter ganhos e perdas separados.

Heath, Chatterjee e France (1995) assinalam, entretanto, que os princípios de contabilidade mental para maximizar satisfação foram derivados e testados em situações sem pontos de referência. Esses autores sugerem que a inclusão de pontos de referência pode fazer diferença. Por exemplo, consumidores provavelmente consideram um desconto de $\$ 5$ (um ganho) mais atraente no contexto de um preço de referência de $\$ 10$ do que no contexto de um preço de referência de $\$ 100$.

A contabilidade mental tem um papel muito importante nas decisões de investimentos financeiros (ROCKENBACH, 2004; SEONGYEON, 2006). Em estudo sobre a teoria dos prospectos e a contabilidade mental, Grinblatt e Han (2005) mostraram que a tendência que alguns investidores têm de supervalorizar suas perdas com ações cria a diferença entre o valor real da ação e seu preço de equilíbrio.

No contexto do cenário descrito na seção introdutória deste trabalho, o consumidor deparava-se com alterações de preços que envolviam um ganho de \$100 e uma perda de \$50 (o preço da cadeira caiu de $\$ 300$ para $\$ 200$ e o preço da poltrona aumentou de $\$ 1.000$ para \$1.050). Nesse cenário, a inclusão do preço de referência na análise cria novas possibilidades de avaliação (HEATH; CHATTERJEE; FRANCE, 1995). Com base no conceito de sensibilidade marginal decrescente da utilidade, justifica-se a sugestão de que talvez o consumidor avalie a situação não em termos absolutos, isto é, um ganho de \$100 e uma perda de $\$ 50$, mas em termos relativos, ou seja, um ganho de $33 \%$ e uma perda de $5 \%$. Conforme 
Heath, Chaterjee e France (1995, p. 91) demonstram, se a avaliação de forma relativa prevalecer, a avaliação separada dos eventos deverá levar a uma satisfação maior do que a avaliação integrada, assumindo-se que o coeficiente de aversão é de 2,5, já que:

$$
\mathrm{u}(33 \%)+\mathrm{u}(-5 \%)>\mathrm{u}(3,8 \%) .
$$

$\mathrm{Na}$ equação acima, o valor de 3,8\% representa o ganho líquido de $\$ 50$, dividido pelo valor inicial de $\$ 1.300$.

Os testes empíricos conduzidos por Heath, Chatterjee e France (1995) deram suporte à proposta de que, em um cenário com dois eventos em que um dos eventos é uma perda e o outro um ganho de maior valor, as pessoas se sentirão melhor se avaliarem os eventos de forma integrada, isto é, pelo ganho líquido, desde que as informações não sejam apresentadas em termos relativos. Quando a apresentação explicitou as diferenças percentuais entre o preço de referência e o preço efetivamente cobrado, os resultados foram alterados e a separação de eventos foi considerada como mais atraente.

Kim (2006) pesquisou o efeito na decisão de compra de se apresentar o preço de forma fracionada ou integral, como, por exemplo, comunicar que determinado produto custa $\$ 140$ já com a taxa de entrega ou falar que esse mesmo produto custa $\$ 120$ e mais $\$ 20$ de entrega. Os resultados mostraram que quanto maior for o valor menor, mais o consumidor irá preferir a opção que apresenta o preço de forma integral. Pesquisando a contabilidade mental no uso de pacotes de preços como tática de vendas de serviços, Gonçalves (2005) colheu evidências de que os pacotes reduzem a percepção de desperdício no caso de o cliente não consumir o serviço comprado.

A contabilidade mental é normalmente caracterizada com o autocontrole dos consumidores para prevenirem gastos em excessos; esse processo mental, porém, é maleável, podendo não funcionar para casos de algumas despesas específicas (CHEEMA; SOMAN, 2006). Duxbury et al. (2005) mostraram que a contabilidade mental funciona quando o consumidor está disposto a poupar dinheiro mesmo que para isso ele tenha que gastar mais tempo, mas o processo não é observado quando o importante é poupar tempo mesmo que para isso se tenha que gastar mais dinheiro. 


\section{PROCEDIMENTOS METODOLÓGICOS}

\subsection{Uma visão geral do estudo}

A metodologia adotada nesta pesquisa seguiu o padrão adotado nas investigações relativas à teoria dos prospectos e contabilidade mental: experimentos com questionários contendo perguntas curtas em situações hipotéticas, em que se apela para a intuição dos participantes do estudo. Thaler (1999) articula os princípios metodológicos associados a essa linha de investigação e argumenta que questões hipotéticas parecem funcionar bem quando os participantes têm acesso a suas intuições e não têm qualquer incentivo em mentir.

O desenho desse experimento seguiu, especificamente, os procedimentos adotados por Thaler (1985) e Heath, Chatterjee e France (1995): os participantes foram solicitados a emitir julgamento sobre a felicidade relativa de dois consumidores fictícios que tinham pela frente situações de compra financeiramente equivalentes. Um dos consumidores lida com um único evento (problema versão integrada), e o outro com dois eventos (versão segregada). Três cenários foram criados para o experimento:

\section{Cenário A}

O Sr. X foi a uma loja comprar uma TV e um videocassete. Ao chegar à loja, entretanto, o Sr. X verificou que, em relação aos preços originais, o total a ser pago pelos dois produtos juntos havia diminuído em 50 reais.

O Sr. Y foi a uma loja comprar uma TV e um videocassete. Ao chegar à loja, entretanto, o Sr. Y verificou que, em relação aos preços originais, o preço da TV havia aumentado em 50 reais e o do vídeo havia diminuído em 100 reais. 
O Sr. X foi a uma loja comprar uma TV e um videocassete. O preço original dos dois produtos, juntos, eram 1.300 reais. Ao chegar à loja, entretanto, o Sr. X verificou que o preço dos dois produtos juntos havia diminuído em1.250 reais.

O Sr. Y foi a uma loja comprar uma TV e um videocassete. O preço original da TV eram 1.000 reais e o do vídeo eram 300 reais. Ao chegar à loja, entretanto, o Sr. Y verificou que o preço da TV havia aumentado em 1.050 reais e o do vídeo havia diminuído em 200 reais.

\section{Cenário C}

O Sr. X foi a uma loja comprar uma TV e um videocassete. O preço original dos dois produtos juntos eram 1.300 reais. Ao chegar à loja, entretanto, o Sr. X verificou que o preço dos dois produtos juntos havia diminuído em $3,8 \%$.

O Sr. Y foi a uma loja comprar uma TV e um videocassete. O preço original da TV eram 1.000 reais e o do vídeo eram 300 reais. Ao chegar à loja, entretanto, o Sr. Y verificou que o preço da TV havia aumentado em 5\% e o do vídeo havia diminuído em 33\%.

A escolha dos produtos (uma TV e um videocassete) obedeceu ao mesmo princípio adotado por Heath, Chatterjee e France (1995): bens complementares, com preços de mercado razoavelmente diferentes, de forma a permitir alterar com facilidade a amplitude dos descontos e aumentos.

Para cada cenário, cada participante do experimento tinha que julgar qual dos dois consumidores ficou mais feliz em uma escala Likert de 5 pontos, que mediu o nível de felicidade relativa entre o $\mathrm{Sr}$. X e o Sr. Y, da seguinte maneira:

\begin{tabular}{|l|l|l|l|l|}
\hline $\begin{array}{l}\text { O Sr. X ficou } \\
\text { muito mais } \\
\text { feliz }\end{array}$ & $\begin{array}{l}\text { O Sr. X ficou } \\
\text { um pouco } \\
\text { mais feliz }\end{array}$ & $\begin{array}{l}\text { Ficaram } \\
\text { igualmente } \\
\text { felizes }\end{array}$ & $\begin{array}{l}\text { O Sr. Y ficou } \\
\text { um pouco } \\
\text { mais feliz }\end{array}$ & $\begin{array}{l}\text { O Sr.Y ficou } \\
\text { muito mais } \\
\text { feliz }\end{array}$ \\
\hline \multicolumn{1}{|c|}{1} & \multicolumn{2}{|c}{3}
\end{tabular}

As instruções aos participantes do experimento salientavam que os eventos eram financeiramente equivalentes e que a preocupação do estudo era avaliar se havia também uma Revista Economia \& Gestão - v. 09, n. 21, set./dez. 2009 

equivalência emocional. Respostas abaixo de 3 dão suporte à versão de integração dos eventos, ao passo que respostas acima de 3 dão suporte à versão de segregação entre eventos. O ponto central da escala representa indiferença entre as duas versões. Finalmente, solicitouse que os consumidores justificassem, em poucas linhas, sua escolha por uma das alternativas da escala.

\subsection{Hipóteses}

O Cenário A descrito na seção anterior define uma situação de controle para o experimento. A inclusão desse cenário visou permitir o teste das propostas de Thaler (1985) de que, em cenários sem preço de referência, a versão com a integração dos eventos seria a alternativa escolhida. Em outras palavras, no Cenário A, a previsão era de que o Sr. X ficaria mais feliz do que o Sr. Y, em razão da proposição de que a integração de uma perda a um ganho de maior valor é mais atraente que a segregação dos eventos. Em termos das hipóteses nas formas nula e alternativa, tem-se então:

$\mathrm{H}_{01}$ : No Cenário A, a média do índice de felicidade relativa será igual a 3.

$\mathrm{H}_{11}$ : No Cenário A, a média do índice de felicidade relativa será significativamente menor que 3 .

O Cenário B inclui a informação sobre o preço de referência e apresenta as alterações do preço final em relação a esse preço de referência em termos absolutos (ganho de \$100 e perda de \$50). Nesse cenário, quem fica mais feliz, o Sr. X ou o Sr. Y? Se o consumidor perceber o problema nos termos descritos (ganhos e perdas em reais), permanece a previsão de que a integração de eventos será preferida e o Sr. X será considerado mais feliz. Se, entretanto, o consumidor perceber as alterações em termos relativos (transformar mentalmente a informação de reais para percentuais), o Sr. Y será considerado mais feliz. Como o cenário descreve a situação em valores absolutos, a hipótese é de que prevalecerá a avaliação em termos absolutos, e a integração de eventos será preferida: 
$\mathrm{H}_{02}$ : No Cenário $\mathrm{B}$, a média do índice de felicidade relativa será igual a 3.

$\mathrm{H}_{12}$ : No Cenário $\mathrm{B}$, a média do índice de felicidade relativa será significativamente menor que 3 .

O Cenário C inclui o preço de referência e apresenta as alterações do preço em termos percentuais (ganho de $33 \%$ e perda de $5 \%$ ), tornando transparente o fato de que, em termos relativos, o desconto no vídeo é alto e o aumento do preço na TV não é. Nesse cenário, a hipótese é de que prevalecerá a avaliação em termos relativos e a segregação de eventos será preferida:

$\mathrm{H}_{03}$ : No Cenário $\mathrm{C}$, a média do índice de felicidade relativa será igual a 3.

$\mathrm{H}_{13}$ : No Cenário $\mathrm{C}$, a média do índice de felicidade relativa será significativamente maior do que 3.

A inclusão da variável nível de escolaridade no estudo se deu em consequência de resultados obtidos na fase-piloto do experimento. A realização de pré-testes foi feita por um período de quatro semanas e incluiu 32 pessoas, com variados níveis de escolaridade. A observação dos resultados dos testes aplicados nessa etapa do projeto criou a impressão de que o grupo de escolaridade mais baixa, sem formação de nível superior, parecia especialmente suscetível a violar o princípio da contabilidade mental de integração dos eventos, quando as alterações de preço eram apresentadas de forma relativa, isto é, o Cenário C. O padrão de escolhas foi de que o Sr. Y estaria sentindo-se mais feliz. A resposta típica para justificar o padrão de escolhas foi de que "com um desconto de 33\%, o Sr. Y estava, é claro, bem melhor que o Sr. X”. Essa tendência não foi observada no grupo com formação de nível superior.

Uma nova pergunta de pesquisa foi então colocada: até que ponto um menor nível de escolaridade influencia o impacto de diferentes maneiras de apresentar alterações de preço? Especificamente, a hipótese foi de que, no caso da versão relativa, a versão integrada dos 
eventos teria um efeito maior no grupo de baixa escolaridade do que no grupo de alta escolaridade.

$\mathrm{H}_{04}$ : No Cenário $\mathrm{C}$, os índices médios de felicidade nos grupos de alta e baixa escolaridades não serão significativamente diferentes.

$\mathrm{H}_{14}$ : No Cenário $\mathrm{C}$, o índice médio de felicidade no grupo de baixa escolaridade será significativamente superior ao índice médio de felicidade no grupo de alta escolaridade.

O experimento contou com duas variáveis independentes: a maneira de apresentar os eventos (versão sem preço de referência, versão com preço de referência e eventos integrados; e versão com preço de referência e eventos segregados) e nível de escolaridade (alto e baixo). A variável dependente do estudo foi a medida de percepção de quem ficou mais feliz: o Sr. X ou o Sr. Y, medida por meio da escala do tipo Likert de 5 pontos.

O experimento teve 180 participantes, distribuídos por seis células. Para fazer parte do grupo de pessoas denominado de baixa escolaridade, foram selecionados 90 alunos do curso de Ensino Médio do SESI do bairro de Laranjeiras no Rio de Janeiro. Participaram alunos da $1^{\mathrm{a}}$ à $4^{\mathrm{a}}$ fase (série) do $2^{\mathrm{o}}$ grau, cuja média de idade foi de 24 anos. $\mathrm{O}$ grupo denominado de alta escolaridade foi composto por alunos da UFRJ, campus do Fundão, média de idade de 22 anos, cursando os mais diversos cursos de graduação daquela Universidade.

Como indica Kerlinger (1980, p. 127), “a força básica da pesquisa experimental está no controle relativamente alto da situação experimental e, em consequência, das possíveis variáveis independentes que possam afetar as variáveis dependentes". As ameaças ao controle são classificadas em questões de validade interna e de validade externa (NACHMIAS, D.; NACHMIAS, C., 1987). A validade interna é dada pelo controle sobre a possibilidade de que outros fatores, que não as variáveis independentes, possam influenciar o comportamento da variável dependente.

A maneira usual de assegurar a validade interna se dá por meio da designação aleatória dos participantes da pesquisa aos grupos experimentais. Esse procedimento foi adotado no caso da variável independente estruturação do problema, mas não foi adotado em relação à variável nível de escolaridade, pela própria característica dessa variável. A discussão sobre a 
PUC Minas E\&G - REVISTA ECONOMIA E GESTÃO

ISSN 1984-6606

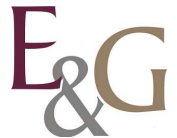

validade externa da pesquisa refere-se à capacidade de generalização dos resultados, tanto para populações maiores do que a amostra que participou do estudo quanto para situações reais de decisão. Em relação a essa questão, Kerlinger (1980, p. 129) comenta:

Em geral os resultados de experimentos de laboratório não podem ser generalizados além do laboratório. Só porque certos resultados foram obtidos em laboratórios não se pode dizer que resultado idêntico ou semelhante ocorrerá fora do laboratório embora possa, e muito bem. Deve-se mostrar, através de pesquisas posteriores, que os resultados se aplicam no campo.

\section{RESULTADOS}

A Tabela 1, a seguir, sumariza os resultados referentes ao índice médio de felicidade para cada célula de pesquisa.

Tabela 1

Índice médio de felicidade

Cenários

\begin{tabular}{c|c|c|c}
\hline $\begin{array}{c}\text { Escolarida } \\
\text { de }\end{array}$ & $\begin{array}{c}\text { Cenário A } \\
\text { (Sem preço } \\
\text { de referência) }\end{array}$ & $\begin{array}{c}\text { Cenário B } \\
\text { (Com preço de referência; } \\
\text { frame absoluto) }\end{array}$ & $\begin{array}{c}\text { Cenário C } \\
\text { (Com preço de referência; } \\
\text { frame relativo) }\end{array}$ \\
\hline Alta & 2,55 & 2,77 & 2,68 \\
Baixa & $(\mathrm{n}=29)$ & $(\mathrm{n}=30)$ & $(\mathrm{n}=31)$ \\
& 2,43 & 2,72 & 3,36 \\
$(\mathrm{n}=33)$ \\
Média Geral & $(\mathrm{n}=28)$ & $(\mathrm{n}=29)$ & 3,03 \\
\end{tabular}

Fonte: Dados da pesquisa

Tabela 2

Estatísticas t

\begin{tabular}{lcccc}
\hline Cenário & $\begin{array}{c}\text { Índice médio } \\
\text { de felicidade }\end{array}$ & $\mathrm{N}$ & Estatística t & Significância \\
\hline $\mathrm{A}$ & 2,49 & 56 & $-3,209$ & 0,001 \\
$\mathrm{~B}$ & 2,74 & 58 & $-1,557$ & 0,0625 \\
$\mathrm{C}$ & 3,03 & 63 & 0,186 & 0,4265 \\
\hline
\end{tabular}

Fonte: Dados da pesquisa 
Conforme a Tabela 2 indica, o índice médio de felicidade associado ao Cenário A, que funcionou como um grupo de controle no experimento, foi significativamente inferior a 3 , confirmando, portanto a primeira hipótese de pesquisa que previa que, em cenários sem a inclusão de preço de referência, os princípios da contabilidade mental propostos por Thaler (1985) seriam confirmados.

A segunda hipótese se refere ao comportamento sob o Cenário $\mathrm{B}$, e a hipótese de pesquisa foi novamente confirmada, embora de forma mais fraca do que foi no Cenário A. A apresentação das alterações em termos absolutos não levou a uma percepção e a comportamentos diferentes daquele observado sob o cenário A. A previsão, consistente com as propostas da contabilidade mental de que a integração mental dos eventos proporcionaria maior satisfação, foi confirmada.

A terceira hipótese se refere à observação do julgamento no Cenário C. A hipótese de pesquisa era de que a versão segregada dos eventos seria escolhida. Essa hipótese, conforme indicado na Tabela 2, não foi confirmada. Uma melhor compreensão do que ocorreu sob esse cenário pôde, entretanto, ser obtida por meio da observação da diferença de resultados entre os grupos de alta e de baixa escolaridade. O padrão de escolhas desses dois grupos foi significativamente diferenciado. Um teste t de diferença de médias foi aplicado aos dados dos dois grupos e mostrou uma diferença significativa nas respostas $(t=-2,093, p=0,04)$. $O$ grupo de alta escolaridade tendeu a escolher a versão integrada de eventos (embora a aplicação do teste t para avaliar se o índice médio dentro da célula era significativamente menor do que 3 não tenha sido conclusivo; $\mathrm{t}=-1,354, \mathrm{p}=0,186)$. Por outro lado, o grupo de baixa escolaridade tendeu a escolher a versão segregada de eventos (embora, novamente, a aplicação do teste t para avaliar se o índice médio dentro da célula era significativamente maior do que 3 não tenha sido conclusivo; $t=1,614, p=0,1116$ ).

A comparação das respostas do grupo de baixa escolaridade nos Cenários B e C indica que o padrão de escolhas dentro do grupo foi diferente entre os dois cenários $(\mathrm{t}=-1,761, \mathrm{p}=$ 0,084). No Cenário $\mathrm{C}$, houve um movimento em direção à versão segregada dos eventos, um comportamento consistente com o que tinha sido observado na fase-piloto do experimento.

De maneira geral, os resultados do experimento deram suporte às hipóteses de pesquisa e corroboraram resultados obtidos nos Estados Unidos. O experimento confirmou as previsões de Thaler (1985) de que, em cenários com ganhos e perdas de menor valor e sem a presença do preço de referência, a versão integrada dos eventos é preferível. 
PUC Minas

A inclusão do preço de referência nos Cenários B e C mudou a preferência pela versão integrada somente no caso do formato relativo de apresentação das informações percentuais (Cenário C) e para o grupo de baixa escolaridade. O suporte empírico para a hipótese de que diferentes maneiras de apresentar alterações de preço podem afetar as maneiras pelas quais essas alterações são percebidas, principalmente para os consumidores de menor grau de escolaridade, é um resultado que vai além das descobertas em pesquisas anteriores.

\section{DISCUSSÃO E CONCLUSÕES}

Os testes empíricos realizados neste estudo confirmaram parcialmente a proposta de Thaler $(1985,1999)$ sobre os princípios de operação da contabilidade mental. Entretanto a maneira de estruturar os preços - formato absoluto ou formato percentual - mostrou-se capaz de alterar percepções e preferências entre alternativas financeiramente equivalentes somente no grupo de participantes com baixo nível de escolaridade. Para esse grupo, a percepção de ganhos e perdas em relação ao preço de referência dependeu da maneira como esses ganhos e perdas foram apresentados.

$\mathrm{Na}$ medida em que esses resultados possam ser estendidos para comportamentos fora do ambiente de laboratório, a conclusão é que a maneira pela qual os consumidores percebem descontos e aumentos em relação a preços de referência depende da maneira como essas alterações de preço são expressas. Descontos percentuais altos podem ter um efeito significativo na percepção dos compradores, mesmo quando o preço de referência for baixo. $\mathrm{Na}$ mesma linha, aumentos percentuais baixos podem ser percebidos como pouco significativos, mesmo quando o preço de referência for alto.

As implicações dessas conclusões para vendedores são imediatas: se o desconto é alto em termos relativos, deve-se apresentar essa informação; caso contrário, não se deve fazê-lo. A lógica se aplica para o caso de descontos descritos em reais. Se o desconto for grande, vale a pena explicitar o valor do desconto; caso contrário, não vale. Nos casos em que tanto o valor percentual quanto o valor absoluto do desconto são altos, provavelmente, a melhor maneira de anunciar a promoção seja explicitando o valor do desconto tanto em termos relativos quanto em termos percentuais (HEATH; CHATTERJEE; FRANCE, 1995). 
A noção de que se pensa em termos relativos é enfatizada pela literatura de tomada de decisão, conforme discutido na Seção 2 deste trabalho. A evidência deste estudo, entretanto, é de que na ausência de uma estruturação relativa, a tendência a pensar em termos percentuais não se manifestou, tanto na presença quanto na ausência de pontos de referência. Uma característica do desenho do experimento pode, contudo, ter limitado a manifestação dessa tendência. Os participantes da pesquisa avaliavam as situações do Sr. A e do Sr. B em um contexto no qual a equivalência financeira era explícita e ressaltada nas instruções. Outrossim, mesmo julgando que o ganho relativo era alto no desconto no preço do videocassete, eles podiam observar que o resultado final era o mesmo para os dois consumidores, e essa percepção pode ter reduzido o efeito de uma percepção inicial, espontânea. Em outras palavras, o desenho do experimento provavelmente trabalhou contra a manifestação espontânea de um pensamento em termos relativos.

Bazerman (2001, p. 499) sugere que as pesquisas têm dado pouca atenção ao consumidor como cliente da pesquisa: "A maioria dos pesquisadores parece ter negligenciado consumidores até mesmo como uma audiência indireta de seu trabalho". Nesse contexto, vale registrar que as situações descritas para os participantes do experimento são similares a promoções frequentemente divulgadas na mídia. Varejistas, por exemplo, em vez de simplesmente fixar determinado preço por um item, incluem na mensagem publicitária um preço de referência. O objetivo é criar a impressão de que o preço efetivamente cobrado representa um ganho em relação ao preço usualmente praticado. Uma construção típica é a "De... Por....". Independentemente da forma, a informação comparativa se caracteriza por apresentar um preço de referência contra o qual o preço real deve ser julgado.

No contexto brasileiro, o debate sobre propaganda comparativa de preços tem sido, aparentemente, inexistente. O tema é, entretanto, relevante; somente no setor varejista brasileiro, os gastos publicitários em 2002 alcançaram a cifra de 2 bilhões de dólares e a estimativa é de que cerca de $50 \%$ desse total se referiu à publicidade associada a preços (GIGLIO, 2004). Como os resultados desse experimento podem contribuir para o debate sobre o tema no contexto brasileiro?

Em primeiro lugar, os resultados do experimento evidenciam que a inclusão de preço de referência em mensagens de preço pode de fato fazer diferença, isto é, influenciar percepções. Os resultados evidenciam ainda que a maneira de apresentar os ganhos e perdas em relação a preços de referência - de forma absoluta ou percentual - também faz diferença. 
Em terceiro lugar, os resultados do estudo sugerem que consumidores de baixa escolaridade são especialmente suscetíveis aos efeitos de diferentes frames

Embora a informação comparativa de preços possa ajudar o consumidor a colocar um valor na oferta que lhe é transmitida e possa resultar em economias significativas de tempo, dinheiro e esforço de busca, essa mesma informação pode também mascarar e criar percepções enganosas sobre a qualidade da transação. Conhecer as expectativas de seus consumidores é extremamente importante para as empresas, já que quanto mais o preço de referência se adequar à expectativa do consumidor, maior será a aceitação do preço no momento da efetivação da compra (CAPORAL; CERETTA, 2004).

\section{LIMITAÇÕES E SUGESTÕES PARA FUTURAS PESQUISAS}

Este estudo apresenta algumas limitações, comuns a pesquisas anteriores sobre o tema (ver, por exemplo, HEATH; CHATTERJEE; FRANCE, 1995). Em primeiro lugar, assumiuse que o preço de referência explicitado na informação dada ao consumidor será o preço de referência efetivamente utilizado. Diversas fontes estão disponíveis ao consumidor para a formação do preço de referência. Nagle e Holden (2002), por exemplo, expandem a discussão sobre formação de preços de referência e sugerem que três categorias de informação, todas parcialmente sob o controle do gestor de marketing, podem se constituir como fonte de preço de referência: o preço corrente a que o comprador está exposto, os preços passados lembrados pelo comprador e o contexto de compra no qual o preço é oferecido.

Uma segunda limitação se refere ao desenho do experimento: além da diferença de escolaridade, outras variáveis (condição econômica, por exemplo) podem ter tido impacto no comportamento da variável dependente do estudo. Não houve utilização de variáveis de controle nesse sentido.

Finalmente, uma terceira limitação se refere ao viés cognitivo adotado nesta pesquisa e em diversos outros experimentos sobre o tema (MAZUMDAR; JUN, 1993; HEATH; CHATTERJEE; FRANCE, 1995;). Outros processos psicológicos estão potencialmente envolvidos nas reações a alterações de preço. Thaler e Johnson (1990), por exemplo, discutem reações afetivas a ganhos e perdas. Urbany Bearden e Weilbaker (1988) sugerem que preços 
de referência exagerados podem criar ceticismo em relação à oferta e reduzir o impacto do preço de referência. O impacto potencial de outras variáveis de natureza psicológica é discutido em diversos artigos sobre o tema (ver, por exemplo, INMAN; McALISTER; HOYER, 1990; GREWAL; COMPEAU, 1999).

\section{Referências}

BAZERMAN, H. Consumer research for consumers. Journal of Consumer Research, v. 27, n. 4, p. 499-504, 2001.

CAMERER, C. Prospect Theory in the Wild: Evidence from the Field. In: Kahneman, D.; Tversky, A. (Ed.) Choices, Values and Frames. Cambridge, University Press, 2000. p. 288300 .

CAPORAL, G.; CERETTA, P. O preço de referência e seu impacto na percepção do consumidor. In: ENCONTRO DE MARKEKING DA ASSOCIAÇÃO NACIONAL DOS PROGRAMAS DE PÓS-GRADUAÇÃO EM ADMINISTRAÇÃO (EMA), 1, 2004. Anais... Porto Alegre: ANPAD, 2004.

CHEEMA, A.; SOMAN, D. Malleable mental accounting: the effect of flexibility on the justification of attractive spending and consumption decisions. Journal of Consumer Psychology, v. 16, n. 1, p. 33-44, 2006.

DAWES, R. Rational choice in an uncertain world. Orlando: Harcout Brace Jovanovich, 1988.

DUXBURY, D.; KESEY, K.; ZHANG, H.; CHOW, S. Mental accounting and decision making: evidence under reverse conditions where money is spent for time saved. Journal of Economic Psychology, v. 26, n. 4, p. 466-580, ago. 2005.

FENNEMA, M. G.; PERKINS, J. D. Mental Accounting Versus Marginal Decision Making: Training, Experience and Justification Effects on Decisions Involving Sunk Costs. Journal of Behavioral Decision Making, v. 21, n. 3, p. 225-359, jul. 2008.

FREITAS Jr., O. A economia no divã. IstoÉ Dinheiro, 13 ago. 2003. Disponível em: http://www.terra.com.br/istoe/1767/1767vermelhas.htm. Acesso em: 6 set. 2007. 
GENDALL, P.; HOEK, J.; POPE, T.; YOUNG, K. Message framing effects on price discounting. Journal of Product \& Brand Management v. 15, n. 7, p. 458-465, 2006.

GIGLIO, E. Os efeitos da credibilidade e do ceticismo sobre a avaliação das propagandas comparativas de preços. Proposta de Tese de Doutoramento, Coppead, Universidade Federal do Rio de Janeiro, 2004.

GONÇALVES, D. A influência dos pacotes de preço na decisão de consumo de serviços. 2005. 113 f. Dissertação (Mestrado em Administração) - Universidade Federal do Rio Grande do Sul, Porto Alegre.

GREWAL, D.; COMPEAU, L. Pricing and public policy: a research agenda and an overview of the special issue. Journal of Public Policy \& Marketing, v. 18, n. 1, p. 3-10, 1999.

GRINBLATT, M.; HAN, B. Prospect theory, mental accounting, and momentum. Journal of Financial Economics, v. 78, n. 2, p. 311-339, 2005.

HEATH, T.; CHATTERJEE, S.; FRANCE, K. Mental accounting and changes in price: the frame dependence of reference dependence. Journal of Consumer Research, v. 22, n. 1, p. 90-97, jun. 1995.

INMAN, J.; McALISTER, L.; HOYER, W. Promotional signal: proxy for a price cut? Journal of Consumer Research, v. 17, n. 1, p. 74-81, jun. 1990.

JONES, S. Implications of behavioral decision theory for health marketing. Marketing Theory v. 7, n. 1, p. 75-91, 2007.

KAHNEMAN, D.; TVERSKY, A. Choices, values, and frames. American Psychologist, v. 39, n. 4, p. 341-350, 1984.

KAHNEMAN, D.; TVERSKY, A. Prospect theory: an analysis of decision under risk. Econometrica, v. 47, n. 2, p. 263-291, mar. 1979.

KAHNEMAN, D.; TVERSKY, A. Reference points, anchors, norms, and mixed feelings. Organizational Behavior and Human Decision Processes, v. 51, p. 296-312, mar.1992. 
KERLINGER, F. Metodologia da pesquisa em ciências sociais: um tratamento conceitual.

São Paulo: EDUSP - Editora da Universidade de São Paulo, 1980.

KIM, H. The effect of salience on mental accounting: how integration versus segregation of payment influences purchase decisions. Journal of Behavioral Decision Making, v. 19, n. 4, p. 381- 391, out. 2006.

MAZUMDAR, T.; JUN, S. Consumer evaluations of multiple versus single price change. Journal of Consumer Research, v. 20, n.3, p. 441-450, dez. 1993.

NACHMIAS, D.; NACHMIAS, C. Research methods in the social sciences. 3. ed. New York: St. Martin's Press, 1987.

NAGLE, T.; HOLDEN, R., The strategy and tatics of pricing: a guide to profitable decision making. 3. ed. New Jersey: Prentice Hall, 2002.

ODEAN, T., Are Investors Reluctant to Realize Their Losses? Journal of Finance, v. 53, n. 5, p.1775-1798, 1998.

ROCKENBACH, B. The behavioral relevance of mental accounting for the pricing of financial options. Journal of Economic Behavior \& Organization, v. 53, n. 4, p. 513-528, abr. 2004.

RUSSO, E.; SCHOEMAKER, P. Decision traps. New York: Ed. Doubleday, 1989.

SEONGYEON, S. Do investors integrate losses and segregate gains? Mental accounting and investor trading decisions. Journal of Business, v. 79, n. 5, p. 2539- 2573, set. 2006.

SERPA, D.; AVILA, M. Percepção sobre preço e valor: um teste experimental. RAE Eletrônica, v. 3, n. 2, jul-dez 2004.

THALER, R. Mental accounting and consumer choice. Marketing Science, v. 4, n. 3, p.199214, 1985.

THALER, R.; JOHNSON, E. Gambling with the house money and trying t break even: the effects of prior outcomes on risky choice. Management Science, v. 36, p. 643-660, jun. 1990. 
THALER, R. Mental accounting matters. Journal of Behavioral Decision Making, v. 12, n. 3, p.183-206, 1999.

THOMAS, M.; MENON, G. When internal reference prices and price expectations diverge: the role of confidence. Journal of Marketing Reseach, v. 44, n. 3, p. 401-409, ago. 2007.

TVERSKY, A.; KAHNEMAN, D. The framing of decisions and the psychology of choice. Science, v. 211, n. 4.481, p.453-458, 1981.

URBANY, J.; BEARDEN, O.; WEILBAKER, D. The effect of plausible and exaggerated reference prices on consumer perceptions and price search. Journal of Consumer Research, v. 15 , n. 1, p. 95-110, 1988.

ZHANG, Y.; FISHBACH, A. The role of anticipated emotions in the endowment effect. Journal of Consumer Psychology, v. 15, n. 4, p. 316-324, 2005. 